\title{
Haití: huRACANES EN UN AÑo de Calma
}

\author{
Haiti: Hurricanes in a year of Calmness
}

\author{
JUAN ESTEBAN MONTES \\ Programa de Chile, Universidad de Notre Dame
}

\author{
ANDREAS FELDMANN \\ Instituto de Ciencia Política, Pontificia Universidad Católica de Chile
}

\section{SANDRA PIRACÉS}

Proyecto Chile-Haití, Centro Internacional de Investigación para el Desarrollo - Instituto de Ciencia Política, Pontificia Universidad Católica de Chile

\section{RESUMEN}

Haití continúa siendo una democracia extremadamente frágil, con capacidades mínimas de ejercer funciones estatales básicas. Haití depende y seguirá dependiendo de la Misión de las Naciones Unidas para la Estabilización de Haití (MINUSTAH) y de la cooperación internacional para mantener su proceso de estabilización política, construcción del Estado, fortalecimiento de la democracia y logro de un desarrollo económico y social sustentable. El gobierno de René Préval, con fuerte apoyo internacional, ha logrado avances importantes en materias de seguridad, planificación y construcción institucional. El año 2008 estuvo marcado por una fuerte crisis de gobierno, gatillada por las alzas en los precios internacionales de los alimentos, severos conflictos con la oposición y desastres naturales causados por las tormentas tropicales y agudizados por la devastación ambiental.

Palabras clave: Haití, Minustah, crisis.

\begin{abstract}
Haiti continues to be an extremely fragile democracy in a state with minimal capacities to perform basic state functions. Haiti depends and will continue depending on the United Nations Stabilization Mission in Haiti (MINUSTAH) and the international cooperation to keep its process of political stabilization, state building, democratic strengthening and achievement of economic and social development to a sustainable level. The René Préval government, with strong international support, has achieved important improvement in security, planning and institutional building. The year 2008 was marked by a deep crisis in the government, triggered by the sudden hike in international food prices, severe conflicts with the opposition and natural disasters caused by tropical storms and aggravated by the environmental devastation.
\end{abstract}

Key words: Haiti, Minustah, crisis. 


\section{INTRODUCCIÓN}

Comparado con los cuatro anteriores, el 2008 fue un año relativamente tranquilo en Haití. No hubo elecciones -no obstante se pospuso la renovación de un tercio del Senado para el 2009-, tampoco grandes novedades en el diseño institucional, ni en el entorno internacional o en la consolidación de la novel democracia. El año estuvo marcado por una súbita alza de los precios de los alimentos y combustibles en abril, que desencadenó protestas que condujeron, a su vez, a la deposición del primer ministro Jacques-Edouard Alexis. Por otro lado, cuatro tormentas que dejaron un reguero de muerte, destrucción y patente evidencia de la debilidad del Estado, además del peligro que implica el desastre medioambiental para la vida de los haitianos.

El cargo de Primer Ministro quedó vacante por más de cuatro meses en una lucha entre el gobierno y el parlamento que es el organismo que debe aprobar su nombramiento. Ante los conflictos desatados por la designación del nuevo Primer Ministro y las posteriores tormentas tropicales, el gobierno logró hacer poco más que sobrevivir y afrontar las crisis. La primera vuelta en la elección parcial de senadores, que se llevó a cabo en abril de 2009 con la exclusión y consecuente boicot del principal partido opositor -Fanmi Lavalás-, resultó en una exigua participación electoral que pone en manifiesto las contradicciones y debilidades del sistema político haitiano. Por una parte, se demostró una cierta voluntad democrática de llevar a cabo las elecciones y, por otra, se comprobó que esa convicción es bastante débil, pues se optó por marginar al principal partido opositor. Esto demuestra la falta de independencia del Consejo Electoral Provisional (CEP), y evidencia la dependencia externa de Haití, pues las elecciones fueron financiadas, resguardadas y controladas por la Misión de Estabilización de las Naciones Unidas en Haití (MINUSTAH) y otros organismos internacionales. Tampoco cabe duda que ni el gobierno ni el sistema democrático de Haití habrían sobrevivido las dificultades del 2008 sin el apoyo de la MINUSTAH y la comunidad internacional en materias de seguridad, economía y administración institucional.

Con todo, hay indicios de ciertos avances en el mediano y largo plazo que podrían considerarse como significativos. En términos de la consolidación democrática, el hecho de que el actual gobierno de Préval y el sistema protodemocrático hayan sobrevivido las dificultades políticas, sociales y económicas del año son signos de alguna fortaleza política y -posiblemente- de legitimidad tanto del gobierno como de la MINUSTAH. Está claro que tanto la MINUSTAH como el sistema democrático nacional se necesitan mutuamente para mantener la estabilidad y viabilidad política y económica de Haití. Ninguno de los dos sobreviviría por sí solo. Por tanto, todavía no es posible anticipar el final de la MINUSTAH sin que se arriesgue seriamente el proceso de reconstrucción haitiano. Sin la presencia de esta misión de las Naciones Unidas, probablemente, no se alcanzarían los niveles actuales de democracia ni subsistencia económica en Haití, pero ésta tampoco sería suficiente recurso como para garantizar el orden y la gobernabilidad en Haití si no hubiera un gobierno democráticamente legitimado.

A partir de agosto del 2008, cuatro tormentas tropicales asolaron gran parte del país. Cientos de haitianos murieron, en la que se convirtió una emergencia humanitaria de proporciones (BBC 2008a). La pobreza, la desigual distribución del ingreso, la falta 
generalizada de servicios básicos, los altos niveles de delincuencia, el analfabetismo, las cárceles hacinadas, el débil sistema judicial, la deforestación extrema y la falta de infraestructura -todos asuntos ya conocidos, pero que no logran ser superados-, continúan demostrando la debilidad del Estado y la continuidad de una historia de inestabilidad política y de erráticas intervenciones internacionales (Feldmann y Montes 2008). En este contexto de estabilidad en el caos, el gobierno de Préval continúa intentando torcer el destino, mientras que la MINUSTAH, que fue establecida en abril del 2004, mantiene su labor de acuerdo con su mandato de velar por la seguridad, la estabilidad política y los derechos humanos en ese país.

No obstante la relativa calma, el 2008 fue un año de grandes dificultades que exigió ajustes para atenuar el impacto de la crisis alimentaria y los desastres naturales que incrementaron la vulnerabilidad de la población y aumentaron la fragilidad del actual gobierno. Por sobre todo, este año acentúa la necesidad de comenzar a responder las muchas preguntas abiertas que pesan sobre Haití. ¿Logrará Préval consolidar su gobierno y poner en marcha procesos de cambio político, económico y social que abran mejores perspectivas para Haití en el mediano y largo plazo? ¿Se están enfrentando los temas de seguridad de manera adecuada, de modo que los sistemas policial y judicial lleguen a garantizar niveles mínimos necesarios de paz y justicia? ¿Hay garantías suficientes de que el sistema político haitiano podría asegurar un juego democrático en las elecciones parlamentarias y comunales del 2009, así como en las presidenciales del 2010? ¿Existe y cuál es el proyecto de crecimiento económico que pueda sacar a Haití de su endémico subdesarrollo y pobreza? ¿Qué rol debe, y puede, seguir cumpliendo la comunidad internacional en Haití? ¿Qué se debe hacer para revertir el daño ecológico de modo que los haitianos puedan gozar de un país sustentable? El 2008 dejó éstas y muchas otras preguntas cruciales sin responder, pero -al menos-creó un mínimo espacio de calma para plantearlas y revalorar su importancia y urgencia.

\section{CRISIS DE GOBIERNO}

No cabe duda que el Presidente René Préval sobrevivió una importante crisis política, económica y medioambiental durante el 2008. Eso, en sí mismo, es un logro significativo. Demuestra que Préval mantiene cierta legitimidad y capacidad de acción. Y viene a confirmar que la oposición, si bien tiene y ejerce capacidad de veto sobre el gobierno, elije algunas veces cooperar y no paralizar completa e indefinidamente el desarrollo político del país. La gobernabilidad en Haití, sin embargo, es ciertamente frágil y, por ahora, dependiente del apoyo internacional, especialmente a través de la MINUSTAH. La crisis de gobierno, los conflictos con la oposición, los problemas para llevar adelante un proceso electoral con forma y tiempo adecuados, el manejo de los desastres naturales, la imposibilidad de despegar de una economía de subsistencia y los problemas continuos en materia de seguridad son todos asuntos que estuvieron presentes durante el 2008 y que demuestran que la capacidad de gobernar en Haití es precaria. La MINUSTAH provee un espacio de seguridad, control, asesoría y logística relativamente estable como para que el gobierno, el parlamento, el sistema judicial y la policía haitianos puedan operar y, a su vez, para 
que los donantes internacionales continúen enviando la vital ayuda económica, en bienes y personal.

Las preguntas inmediatas, más difíciles e interesantes de responder, entonces, son si el gobierno y el sistema político y económico salieron fortalecidos o debilitados de la crisis y si serán capaces de enfrentar los desafíos del futuro inmediato. ¿Fue el 2008 el inicio de un período de mayores problemas, posiblemente terminales, para la nueva democracia haitiana o, más bien, fue un año duro pero de aprendizaje y consolidación? Durante el 2009 vendrán más pruebas difíciles en el ámbito político-administrativo con elecciones parlamentarias complejas, la crisis económica internacional que afectará a los países donantes tanto como a Haití, una reforma constitucional incierta y la esquiva consolidación de la reforma al sistema de seguridad.

El 14 de mayo de 2009 se cumplió el tercer aniversario de la designación de René Préval como Jefe de Estado de Haití, lo que es de por sí un signo de estabilidad. Aún le restan, sin embargo, dos años para completar su mandato, los que ciertamente no pueden darse por descontado. Según miembros de organizaciones civiles haitianas, la condición de vida del pueblo no ha mejorado desde la llegada de Préval (Inter American Dialogue 2007), salvo en materia de seguridad. El hambre y la carencia de servicios sociales e infraestructura, sumados a la violación sistemática de los derechos socioeconómicos y políticos de la oposición, son males que persisten pese al esfuerzo local y de la comunidad internacional por contrarrestarlas (Human Rights Watch 2009). Las expectativas insatisfechas y el afán por sacar adelante su gobierno, a casi cualquier costo, están pasando la cuenta a Préval. Sin embargo, ha habido avances en materia de seguridad, con la consolidación de la Policía Nacional Haitiana (PNH) y en cuanto a la capacidad local para diseñar modelos institucionales y planificación de base en políticas públicas (seguridad y justicia, medio ambiente, desarrollo económico).

La opinión generalizada de que la administración del ex Primer Ministro Jacques-Edouard Alexis era poco efectiva en lo económico, le costó el cargo al ser censurado por el Senado el 12 de abril de 2008. Se le criticó la poca capacidad para controlar el alza de los precios de los alimentos y combustibles, los que venían escalando por meses y que terminaron desencadenando violentas protestas a comienzos de abril. El Presidente Préval también fue criticado por su falta de liderazgo, pero cuando habló al país, tres días después de iniciadas las manifestaciones de abril, éstas se calmaron, demostrando su ascendencia y apoyo. Hay que admitir que a ello ayudó también una acción más decidida de las fuerzas militares de las Naciones Unidas (BBC 2008b). Demostrando, una vez más, que -tanto el gobierno haitiano como la MINUSTAH- se necesitan mutuamente para asegurar mínimos de gobernabilidad.

Durante cuatro meses el presidente intentó infructuosamente lograr un acuerdo con el parlamento para nombrar a un nuevo Primer Ministro. Hay evidencia de que las protestas de abril, a causa del alza de los precios, habrían sido -si no instigadas-, al menos, aprovechadas y aumentadas por la oposición. Algunos sectores insisten en explotar políticamente la pobreza y la desesperación relacionándolas con traficantes de drogas, remanentes de las bandas delictuales y comerciantes que se benefician del caos (BBC 2008c). A raíz de la crisis 
política, se pospusieron la reunión de países donantes y la elección de más de un tercio de senadores que debía haberse efectuado en abril del 2008 (ya había sido postergada durante el 2007). Eso implica que hasta junio del 2009, cuando se efectúe la segunda vuelta para la elección de los 12 senadores, la Cámara Alta deberá continuar funcionando con sólo 18 de sus 30 miembros. En este ambiente de incertidumbre y fricciones políticas entre el gobierno y la oposición, negociar la formación de un nuevo gobierno resultó difícil. Dos candidatos fueron rechazados por el parlamento, hasta que Michèle D. Pierre-Louis (61) recibiera la aprobación de ambas cámaras del parlamento haitiano y pudiera asumir su cargo el 5 de septiembre de 2008 (International Crisis Group 2008a).

Pierre-Louis es especial no tan sólo por ser la segunda mujer premier, sino también por su cercanía con el actual mandatario y por su reputación de ser una proactiva líder en organizaciones comunitarias, así como en instituciones públicas y privadas. Esta economista formada en Nueva York, con estudios de gestión de aeropuertos en Francia, entre 1995 y 2008 encabezó Fokal, una organización no gubernamental financiada por el magnate estadounidense George Soros. Más relevante, ella viene con una experiencia de 30 años de trabajo con jóvenes, mujeres y campesinos, ámbitos centrales de la problemática haitiana. Pierre-Louis llegó al cargo con la misión de diseñar un programa de gobierno que ataque los problemas de la nación. Para ello pidió plazo hasta febrero del 2009, sabiendo que la tarea de estructurar un plan comprensivo y una estrategia política y económica capaces de iniciar un camino de consolidación política y desarrollo sería altamente compleja. A fin de generar estabilidad y gobernabilidad, Pierre-Louis mantuvo siete ministros de la administración anterior y ha hecho esfuerzos por mejorar la comunicación con el parlamento (Brea 2008).

El 2 de diciembre de 2008 presentó un completo plan de acción basado en un esquema nacionalmente integrado y coordinado, para el desarrollo de infraestructura y generación de empleos, metas de igualdad, formación de capital humano, recuperación del medio ambiente y fortalecimiento de la seguridad y la justicia. Si bien al comienzo y en gran medida hasta ahora (mayo de 2009) Pierre-Louis ha recibido un fuerte apoyo de sus connacionales y de la comunidad internacional para asumir como Primera Ministra, hasta el momento no ha podido generar los acuerdos ni acceder a los recursos necesarios para adoptar las medidas que resuelvan las urgentes necesidades de los haitianos. Más aún, ha sido criticada por el débil manejo de los efectos de los huracanes (Médecines Sans Frontièrs 2008). El relativo apoyo a Pierre-Louis y a Préval hace pensar que se refuerzan mutuamente y que, de esta manera, generan un gobierno más confiable.

\section{LA OPOSICIÓN NO DA TREGUA: LOS COSTOS DE UN SISTEMA DE PARTIDOS ULTRAFRÁGIL}

Partiendo por la censura de Alexis, siguiendo con el prolongado rechazo a aprobar su reemplazante, la fuerte crítica al manejo de la crisis humanitaria ocasionada por los huracanes, el bloqueo del presupuesto -a inicios del 2009-y el boicot de Fanmi Lavalás a las elecciones de un tercio del Senado en abril de 2009, la oposición ha asumido una actitud 
Tabla 1: Composición del Gabinete de la Administración de René Préval (septiembre, 2008)

\begin{tabular}{ll}
\hline Cartera & \multicolumn{1}{c}{ Personero $^{\text {a }}$} \\
\hline Primera Ministra & Michèle Pierre-Louis \\
Asuntos Exteriores y Culturales & Charles Manigat \\
Haitianos en el Extranjero & Paul-Antoine Bien-Aimé \\
Economía y Finanzas & Daniel Dornsainvil \\
Planificación y Cooperación Externa & Jean Max Bellerive \\
Agricultura, Recursos Naturales y Desarrollo Rural & Joanás Gue \\
Turismo & Patrick Delatour \\
Comercio e Industria & Marie Josée Garnier \\
Trabajo Público, Transportes y Comunicaciones & Gérald Jean-Baptiste \\
Medio Ambiente & Jean-Marie Claude Germain \\
Educación Nacional y Formación Profesional & Joël Desrosiers Jean-Pierre \\
Juventud, Deportes y Acción Cívica & Evans Lescouflair \\
Salud Pública y Población & Alex Larsen \\
Asuntos Sociales y Trabajo & Gabrielle Beaudin \\
Género y Derechos de la Mujer & Marie-Laurance Jocelyn Lassègue \\
Cultura y Comunicación & Olsen Jean Julien \\
Justicia y Seguridad Pública & Jean-Joseph Exumé \\
Ministro Delegado Encargado de Relaciones con el Parlamento Joseph Jasmin \\
\hline
\end{tabular}

Fuente: Presse Francophone.

desafiante frente al Ejecutivo durante el último año. Por una parte, esta es una buena noticia. No hay democracia sin oposición, tal como Robert Dahl argumentara hace más de cuatro décadas (Dahl 1972). Por otro lado, la oposición haitiana es institucionalmente débil y fraccionada, pero controla un poder de veto parlamentario y de movilización social que no duda en ejercer a ultranza si le parece adecuado. Así, algunos sectores de la oposición se dedican a hacer ingobernable el país, si bien es cierto que no toda la oposición es puramente obstruccionista, como lo demostró la aprobación de la Primera Ministra, su plan de desarrollo y de algunas leyes importantes como las de reforma del sistema judicial. Pero el gobierno no cuenta con apoyo mayoritario en el parlamento y cada iniciativa debe ser negociada con múltiples actores. Este tipo de oposición puede terminar minando la legitimidad, no sólo del gobierno -aunque carezca de capacidad de levantar uno alternativo-, sino también de la frágil democracia haitiana y de su devastada economía (International Crisis Group 2008a).

El gobierno de Préval partió con el apoyo mayoritario de una coalición informal de partidos que ha ido disminuyendo su apoyo progresivamente, aunque Lespwa (La Esperanza), su partido político, se ha fortalecido en cada elección complementaria. El sistema de partidos haitianos es extremadamente débil, fragmentado, personalista, con baja disciplina partidaria, 
frecuentes divisiones y cambios de partido por sus parlamentarios. Es de lo más opuesto que uno pueda encontrar en un sistema institucionalizado conforme a los conceptos de Mainwaring y Scully (1995). Por su parte, la implementación del sistema electoral adolece de grandes deficiencias: Depende del financiamiento y apoyo logístico de la MINUSTAH, la OEA y otros organismos internacionales; la PNH no tiene capacidad de vigilar las elecciones, las que son frecuentemente diferidas, mientras que el Consejo Electoral Provisorio (CEP) no garantiza imparcialidad, pues es influenciado por el gobierno. Actualmente, hay 17 partidos con representación en la Cámara de Diputados, pero se habla de la existencia de cerca de cien partidos en Haití, en donde los líderes crean su propia bancada casi sin restricciones y, de la misma manera, la hacen desaparecer (Hyppolite Pierre 2009).

A fines del 2008 la oposición dio por concluida la luna de miel de Pierre-Louis, reclamando que los 197 millones de dólares del programa PetroCaribe (por el cual Venezuela transfiere petróleo a bajo costo al Estado haitiano el que lo vende a precio de mercado a los distribuidores para generar un fondo de ayuda) que ella usó para la limpieza y reconstrucción luego de las tormentas, no fueron bien empleados y que, casi al comenzar la nueva temporada de huracanes, aún no se resuelven los problemas del año anterior. Se le acusa de no ser suficientemente firme frente a Préval, quien tiene reputación de meterse en todo, y se le critica no haber sido capaz de crear un apoyo sólido en el parlamento (Louidor 2009).

No es fácil esclarecer cuáles son las razones para la obstrucción de la oposición, pero no cabe duda que el frágil, fraccionado y personalista sistema de partidos cumple un rol clave. Ciertamente, hay razones institucionales que ayudan a explicar el tipo de oposición que hay en Haití, como la carencia de una ley de partidos políticos, un sistema electoral que favorece una multiplicidad de bandos débiles y una constitución altamente cuestionada. Por otra parte, hay fenómenos políticos históricos -como largos períodos autoritarios y repetidas intervenciones extranjeras- que generan desconfianzas políticas profundas. También el hecho de que casi todos los partidos sólo tengan unos pocos años de existencia (Lavalás y Organisation du Peuple en Lutte (OLP) son de los más antiguos y surgieron recién a mediados de la década de los 90) o las débiles raíces de los partidos en la sociedad, la frecuente manipulación de los partidos y las elecciones por líderes personalistas. A todo esto se suma una fuerte polarización en torno a la figura del ex Presidente Jean-Bertrand Aristide y a la intervención internacional. Pero también hay razones políticas más inmediatas. Hay quienes consideran que la razón de la falta de apoyo radica en el interés de los parlamentarios por obtener mayor financiamiento para sus distritos, así como prebendas personales. Pero también -según la opinión de dirigentes de organismos para el desarrollo, entre otrosla férrea oposición responde a que "el gobierno sigue aplicando una política neoliberal que le dictan los organismos de financiamiento internacional. Los ejes fundamentales de esta política no giran en torno a la mejora de la calidad de vida de la población, sino en el mantenimiento de la estabilidad de los indicadores macroeconómicos y en la reducción de los gastos sociales" (Louidor 2009). De esta manera, los esfuerzos gubernamentales se enfocarían en satisfacer las demandas estructurales de los organismos internacionales y en acciones particulares para asuntos específicos, a modo de microproyectos, que no contribuyen a mejorar la calidad de vida de la población en su totalidad. 
Debemos recordar que tanto el apoyo al gobierno como a la oposición están conformados por múltiples, débiles e indisciplinados partidos, en los que muchas veces los parlamentarios se cambian de bando o actúan en forma libre, además de no tener siempre la capacidad ni el apoyo adecuados para cumplir a cabalidad sus funciones legislativas y fiscalizadoras. Más allá de su debilidad, sin embargo, los partidos tienen la capacidad para convocar protestas masivas y bloquear la legislación en el parlamento. Fanmi Lavalás continúa teniendo un fuerte apoyo entre la población más pobre, pero -luego de su inicial apoyo a Préval en las elecciones de 2006- pasó a formar parte de una activa oposición (Shamsie y Thompson 2006). Las recientes y masivas protestas (se estiman 20.000 las personas que asistieron) convocadas por Lavalás el 28 de febrero de 2009, para conmemorar el quinto aniversario del golpe y salida de Aristide, se repitieron días después a raíz de la visita del ex presidente Bill Clinton y del Secretario General de las Naciones Unidas, Ban Ki-Moon, a quienes solicitaban favorecer el retorno del ex Presidente Jean-Bertrand Aristide. Además, las protestas respondían al enojo de los partidarios de Fanmi Lavalás por la exclusión por parte del CEP, controlado por Préval, de todos sus candidatos para las próximas elecciones senatoriales de abril de 2009. Los argumentos del CEP para excluir a los candidatos de Lavalás fueron meras excusas formales, las que aprovecharon las divisiones internas en Lavalás para dejarlos al margen de la elección. El gobierno de Préval, incluso, destituyó a un juez que sentenció la necesidad de incluir las candidaturas de Lavalás (Pina 2009).

El gabinete del ex Primer Ministro Alexis era apoyado por seis partidos, de los cuales sólo tres continúan durante la gestión de Pierre-Louis. ${ }^{1}$ El mismo Alexis y sus partidarios en el parlamento se sintieron ofendidos por su salida del gobierno y pasaron a engrosar la oposición. Incluso estuvieron entre los que bloquearon los dos primeros nombres propuestos por Préval para el cargo de Primer Ministro. El Senado perdió cuatro senadores del partido oficialista Lespwa: Dos que cumplieron su período junto a otros 8 senadores, un tercero que murió y uno más que fue inhabilitado. Los 12 han estado sin reemplazo debido a la postergación de las elecciones que debían haberse celebrado en noviembre de 2007, fueron postergadas para abril de 2008 y, luego, para abril del 2009. Estos continuos aplazamientos de las elecciones en Haití son un mal indicio para la consolidación de la democracia (Pina 2009). Los partidos que apoyan el gobierno de Préval suman 52 diputados, de un total de 99 asientos. Pero la falta de disciplina partidaria, el oportunismo político y el personalismo que plaga el sistema de partidos no permite asegurar un apoyo consistente para el gobierno.

A comienzos del 2009 Préval, Pierre-Louis y el presidente del Senado aparecieron haciendo un urgente llamado a la unidad y cooperación entre el gobierno y la oposición. Esta cooperación se hace indispensable para aprobar el presupuesto de 2009-2010, pero su discusión fue suspendida por el Senado y luego por la Cámara de Diputados a fines del 2008, reclamando la eliminación de un impuesto a las telecomunicaciones y a los pasaportes y mayor claridad en el uso del fondo de emergencia.

1 Lespwa, Fusion des Sociaux Démocrates, Organisation du Peuple en Lutte, Alyans, Union y Fanmi Lavalás estaban en el gobierno de Alexis. Lavalás no negoció para integrarse al nuevo gobierno. OPL y Alyans negociaron, pero se retiraron del gobierno. 
Las reformas constitucionales en materia de doble nacionalidad para incentivar la participación de los haitianos en el extranjero, así como las reformas para generar mayor estabilidad política y seguridad, incluyendo el conflictivo tema de cómo incorporar a los ex miembros de las fuerzas armadas, institución abolida en 1994 tras el golpe de 1991 contra Aristide, anticipan mayores controversias entre el gobierno y la oposición para el resto del 2009. La oposición reclama que la reforma constitucional también apuntaría a asegurar la posibilidad de reelección de Préval en noviembre de 2010. Lo mismo se verá en torno a las elecciones de diputados, un tercio del Senado, autoridades municipales y locales, previstas para noviembre del 2009.

La elección de 12 senadores, recién celebrada el 19 de abril de 2009, ya ha resultado en masivas manifestaciones, acusaciones de exclusión injustificada de Lavalás por el CEP, y en el consecuente boicot. La exigua participación electoral, que habría llegado sólo al $11 \%$ de los 4,5 millones de potenciales electores, junto a la dispersión de votos entre los muchos candidatos y partidos, hizo necesario que haya una segunda vuelta para todos los asientos senatoriales en disputa, la que debería tener lugar el 7 de junio próximo. Si estas elecciones son un buen anticipo de lo que sucederá en esa segunda vuelta, como en las elecciones parlamentarias y municipales de fines de 2009 y las presidenciales de 2010, los auspicios para la continuidad democrática, incluso conforme a definiciones formales mínimas, son -al menos- frágiles. La comunidad internacional continúa siendo un actor fundamental en el manejo, financiamiento y control de las elecciones en Haití.

\section{DESAFÍOS DE UNA FRÁGIL ECONOMÍA}

La economía haitiana ha tenido buenos resultados moderados en estos cuatro años con presencia de la MINUSTAH. Los pronósticos para el 2008 eran bastante más auspiciosos, pero el alza internacional de precios de los alimentos y los huracanes hicieron que la inflación alcanzara el 10,1\% y la tasa de crecimiento de la economía llegara sólo al 1,5\%. Con todo, la situación económica de Haití sigue siendo desalentadora, ya que presenta una extrema pobreza de magnitudes. Un tercio del PNB, unos 1,65 billones de dólares americanos (2006), proviene de remesas de los haitianos en la diáspora, especialmente de EE.UU., Canadá y Francia, tres países que muy posiblemente se verán afectados por la crisis económica internacional. En Haití, 1,1 millones de personas dependen directamente de las remesas internacionales para sobrevivir. La economía haitiana sigue dependiendo fuertemente de las donaciones internacionales en dinero y en especies, las que a su vez dependen de la continua presencia de la MINUSTAH que garantiza la estabilidad, seguridad, asesoría y control al gobierno, que son alicientes para que los actores internacionales estén dispuestos a efectuar donaciones a Haití. El presupuesto de 256 millones de dólares de 2009 es financiado en un 60\% por donantes extranjeros. En otros años las cifras han sido similares. Del presupuesto público, 130 millones de dólares van para la Policía Nacional de Haití. La reciente cumbre de donantes organizada por el BID reunió el compromiso de US\$ 324 millones adicionales para los próximos dos años, de los cuales 40 millones se incorporarán al presupuesto del 2009. La mayor parte de las donaciones no van directamente al gobierno haitiano sino que se ejecutan a través de ONG's, la MINUSTAH, 
iglesias y otras instituciones privadas. Sin embargo, es usual que una parte significativa de las ofertas de las donaciones no llegue a concretarse. La capacidad exportadora de Haití continúa siendo muy débil, no logra alcanzar sostenidamente los 500 millones de dólares al año y está basada en un par de productos agrícolas, artesanía y pequeñas manufacturas (CEPAL 2008, BID 2008).

Entre las novedades del 2008 estuvo la puesta en marcha de la Estrategia Nacional para el Crecimiento y la Reducción de la Pobreza (Stratégie Nationale pour la Croissance et la Réduction de la Pauvreté), aprobada en noviembre del 2007 por el Ministerio de Planificación y Cooperación Externa de Haití junto con colaboradores internacionales. El documento, con el que se han comprometido los cooperantes internacionales y la autoridad haitiana, define los principales lineamientos para actuar por el desarrollo de la nación, destacando la implementación y mejora de los servicios básicos. Tiene como metas mejorar la gobernabilidad y fortalecer la seguridad y el sistema de justicia. Para ello promueve cuatro vectores de crecimiento: La agricultura y el desarrollo rural, el turismo, la infraestructura y la ciencia y tecnología, todo ello dentro de un marco de estabilidad macroeconómica y prácticas de buen gobierno y transparencia (Ministère de la Planification et la Cooperation Externe de Haiti 2009).

El año 2008 registró un desaceleramiento en la economía en comparación con los tres años anteriores. Esto se explica por la crisis mundial que provocó el alza de los precios de los alimentos y los combustibles en Haití en aproximadamente $40 \%$. En seis meses el precio internacional del arroz aumentó en un 60\% y el del maíz en un 90\% (International

Tabla 2: Exportaciones brutas anuales de Haití según productos. En millones de dólares americanos

\begin{tabular}{|c|c|c|c|c|c|c|}
\hline Productos & 2003 & 2004 & 2005 & 2006 & 2007 & 2008 \\
\hline Café & 3,55 & 4,28 & 3,78 & 4,13 & 2 & 3,74 \\
\hline Cacao & 6,05 & 6,51 & 6,91 & 7,35 & 6,17 & 7,43 \\
\hline Aceites esenciales & 5,08 & 5,86 & 7,15 & 4,94 & 13,13 & 17,79 \\
\hline Pita y cordel & 1,58 & 0,95 & 1,18 & 0,87 & 0 & 0 \\
\hline Mango & 4,66 & 6,63 & 8,92 & 9,45 & 7,93 & 10 \\
\hline Langosta & & & & & 4,33 & 5,16 \\
\hline Otros productos primarios & 9,1 & 8,95 & 5,32 & 2,24 & 0,89 & 0,85 \\
\hline Pequeña industria & 4,94 & 4,33 & 5,44 & 7,32 & 5,16 & 2,59 \\
\hline Artículos de manufactura & 108,44 & 124,84 & 154,72 & 169,66 & 180,58 & 165,07 \\
\hline Otros productos industriales & 7,46 & 6,76 & 6,23 & 2,14 & 0,67 & 1,24 \\
\hline Ajustes por evaluación & 12,68 & 13,91 & 17,17 & 17,82 & 18,85 & 18,31 \\
\hline Total & 163,55 & 182,43 & 216,82 & 225,92 & 239,7 & 232,18 \\
\hline Ajustes por clasificación & 169,62 & 195,26 & 242,02 & 265,32 & 282,44 & 258,19 \\
\hline Total Export. & 333,16 & 377,69 & 458,84 & 491,24 & 522,14 & 490,38 \\
\hline
\end{tabular}

Fuente: Banco de la República de Haití. 
Crisis Group 2009a). A partir del 7 de abril de aquel año el precio del transporte aumentó rápidamente en un 50\%, ante lo que muchos "tap tap" de transporte colectivo debieron dejar de circular, y se agudizaron los asaltos a bombas de bencina (cerca de 22 gasolineras fueron asaltadas durante ese periodo (Gauthier 2008).

La negativa inicial del gobierno haitiano para tomar medidas paliativas se tradujo en disturbios y protestas frente al Palacio Nacional en Puerto Príncipe, las que condujeron a la destitución del Primer Ministro, ya mencionado. Al menos seis personas resultaron muertas producto de las manifestaciones. La semana de parálisis económica que hubo a raíz de la crisis del alza de costos, significó una pérdida aproximada de \$100 millones de dólares en costo directo para el sector privado, merma que el gobierno no compensará y que desincentiva a potenciales inversores (BBC 2008b).

En un país en que el $54 \%$ de la población vive en situación de extrema pobreza -con menos de US\$ 1 estadounidense al día-y un 78\% con menos de 2 dólares estadounidenses diarios, la mayoría de la población tiene como principal gasto la alimentación, por lo que un aumento de los precios de los commodities afecta su supervivencia (Banco Mundial 2007). Los subsidios gubernamentales a productos derivados del petróleo y los alimentos ${ }^{2}$ no fueron suficientes para atenuar los reclamos de un pueblo con problemáticas sociales históricamente insatisfechas. Aparte de la alimentación, otros servicios fundamentales tampoco están -ni mínimamente-cubiertos. Sobre un 70\% de la población carece de acceso a salud, mientras que la educación, el agua potable y la electricidad siguen siendo un privilegio. El alto índice de extrema pobreza no impide que Haití tenga uno de los peores indicadores de distribución de la riqueza en el mundo -un coeficiente Gini de 0,65- y el lugar 148 entre 179 países en el Índice de Desarrollo Humano (PNUD 2008).

Ante el aumento del número de secuestros y la inestabilidad política de Haití, la comunidad internacional reaccionó haciendo un llamado a la ayuda. En mayo, el Banco Mundial anunció el envío de alimentos a la isla por un total de 30 millones de dólares americanos. Por otra parte, algunos países como Brasil, se comprometieron a aumentar sus contingentes militares. La vulnerabilidad de la población es extrema y la falta de soluciones efectivas en la cobertura de las necesidades básicas de la población puede resultar en mayores disturbios políticos en un futuro cercano.

Según datos del Ministerio de Economía y Finanzas de Haití, la deuda externa a diciembre del 2008 era de 1.853,2 millones de dólares estadounidenses, mientras que 2/3 de la población sigue desempleada o subempleada. Muchos haitianos carecen de más opción que dejar el país, muchas veces en viajes marítimos riesgosos, para probar destino en otra nación con una situación irregular, y asegurar su subsistencia y la de sus parientes en la isla.

\section{DESASTRES NATURALES: LA BOMBA DE TIEMPO MEDIOAMBIENTAL CONTINÚA EXPLOTANDO}

Durante los meses de agosto y septiembre, cuatro tormentas tropicales y huracanes (Fey, Gustav, Hanna e Ike) asolaron las costas y, principalmente, la zona central de Haití

2 La regalía a los importadores de arroz fue de un $15 \%$ por saco. 
(Gonaïves), ocasionando cerca de 800 muertes (las inundaciones de 2004 ya habían causado otras 3.000 muertes), 310 desaparecidos, 112.000 viviendas dañadas o destruidas, y cerca de un millón de afectados -más de un décimo de la población- por los destrozos en la agricultura y la infraestructura. Estos desastres naturales han venido a confirmar que la crisis medioambiental debido a la deforestación extrema del país constituye una bomba de tiempo que no solamente amenaza la integridad física de los casi diez millones de haitianos, sino que periódicamente estalla con consecuencias humanitarias, económicas y políticas desastrosas. La topografía haitiana, con cadenas montañosas que alcanzan los 2.700 metros sobre el nivel del mar, y laderas empinadas y deforestadas, deja poca superficie cultivable y congrega a la población en la peligrosa base de los valles. Su ubicación geográfica, en zonas de fuertes lluvias y huracanes, y la deforestación, han causado sobre 19.000 muertes desde 2001, la destrucción de cerca de 200.000 casas y pérdidas por más de US $\$ 5$ billones (International Crisis Group 2009b).

Las inundaciones dañaron seriamente la agricultura (menos del 2\% de la cubierta forestal subsiste en Haití) y la infraestructura, agravaron la escasez de alimentos y provocaron un alza de la inflación que empujó a aún más haitianos a la condición de pobreza. Con posterioridad a las tormentas, se estima que un tercio de la población está en condición de necesitar ayuda alimenticia (Coordination Nationale de la Sécurité Alimentaire 2008). Las pérdidas económicas de estos desastres naturales sumaron 1.000 millones de dólares americanos, equivalentes al 15 por ciento del producto interno bruto de Haití. Ante esta situación de desborde, el llamado a la comunidad internacional a preocuparse por la situación haitiana aumenta. Pero la crisis financiera mundial está haciendo difícil a los donantes cumplir con los compromisos para reducir la diáspora (International Crisis Group 2009a).

La crisis medioambiental va mucho más allá que las inundaciones y los huracanes. La falta de fuentes energéticas es crónica. Un $5 \%$ de la energía viene de la hidroelectricidad, otro $20 \%$ viene del petróleo, dejando el $75 \%$ restante al carbón vegetal. Cabe mencionar que la deforestación destruye los suelos cultivables, reduce la posibilidad de captar agua potable (después de las inundaciones de 2008 se redujo de $40 \%$ a 10\% la disponibilidad de agua potable en el Departamento del Sureste), genera enormes depósitos de barro y fomenta la emigración desde el campo a la ciudad. Por esto, se calcula en 75.000 las personas que llegan cada año a Puerto Príncipe a engrosar los campamentos, el hacinamiento y el desempleo. Estas condiciones generan a su vez grandes riesgos para la salud pública (International Crisis Group 2009a).

Las políticas medioambientales del gobierno no alcanzan a implementarse y se limitan a la limpieza de las ciudades después de las inundaciones, cuando la base de los problemas está en la deforestación de las montañas y el campo. La ayuda internacional ha intentado llenar el vacío de políticas públicas, pero tampoco hay un plan de acción internacional coordinado y comprensivo en la materia, por lo que los proyectos son de bajo impacto sistémico. La crisis medioambiental se transforma así en un tema central con serias consecuencias para la paz y la seguridad (International Crisis Group 2009b).

Otro desastre de magnitudes sucedió en noviembre, una vez pasados los huracanes. Entonces, la estructura de una escuela en Puerto Príncipe sufrió un colapso, mientras 500 
alumnos y profesores hacían clases dentro de ella. Este evento mostró la vulnerabilidad de la infraestructura haitiana, una realidad difícil de esconder y que cruza cada ámbito del quehacer nacional.

Muchas iniciativas, de corto y largo plazo, son necesarias para enfrentar la devastación ambiental; el problema radica en el conflicto que generan las soluciones a largo plazo, con altos costos para la población y -por lo mismo- con costos políticos inmediatos. Lo que es claro es que no basta con limpiar los efectos de los huracanes y con profundizar los canales y barreras de contención de los cauces. Más bien, es necesario enfrentar temas de propiedad de la tierra, forestación con especies mayores que den frutos en algunos años, como mangos y otros frutales o madera para mueblería y construcción, generar mejor calidad de vida en las zonas rurales, incentivar el uso de fuentes de energía diferentes al carbón vegetal, entre otros.

\section{VI. ¿QUÉ PASA CON LA TAN ESPERADA REFORMA DE LA SEGURIDAD?}

En vistas a construir capacidad estatal mínima, que controle el uso legítimo de la violencia y expanda el precario imperio del derecho (y, eventualmente, facilite el turismo) es indispensable avanzar más rápido en la largamente anunciada reforma de la seguridad. El plan aprobado en el 2006 incluye reformas al sistema judicial, a la Policía Nacional Haitiana $(\mathrm{PNH})$ y al sistema penitenciario. Cada uno de estos sectores continúa presentando desafíos ineludibles que en conjunto deben enfrentar el control de las bandas delictivas, el narcotráfico, los secuestros, el control de la porosa frontera con República Dominicana y del borde costero y el manejo de las protestas en las ciudades (HRW 2008).

Retrocediendo en el tiempo, durante el 2007 la situación en materia de seguridad mejoró visiblemente con el aumento del número de oficiales de la PNH que surgieron al alero de las fuerzas de paz de las Naciones Unidas. Los barrios, antiguamente dominados por bandas de narcotraficantes, como Citè Soileil y Martissant, fueron liberados de estos yugos. Cerca de 750 miembros de bandas armadas fueron detenidos en ese tanto. Y, aun cuando el número de secuestros disminuyera durante el 2007, al año siguiente hubo nuevos asaltos y los secuestros se expandieron a otros objetivos, especialmente niños de escuelas, lo que aumentó la sensación de inseguridad entre la población. Los 9.000 policías en servicio aún requieren capacitación en el control de fronteras, inteligencia, control de manifestaciones, manejo de secuestros y narcotráfico, pero, sobre todo, deben ser incrementados a -por los menos- los 14.000 que se planea deben haber en el 2011.

La formación de policías es un ámbito en que la comunidad internacional puede cumplir un rol mucho más activo. Hace falta formar más y mejores oficiales con capacidad de mando y liderazgo. La falta de cuarteles policiales, de transporte, de uniformes y materiales es seria. Además, es vital incorporar a más mujeres para atender mejor a las víctimas de ese género y a niños; la meta de 30\% del contingente está muy lejos aún. El proceso de revisión de antecedentes y veto de los policías envueltos en casos de corrupción y crimen va lento y presenta muchas dificultades como falta de capacidad administrativa, protección de los colegas y faltad de decisión para despedir a los involucrados. El fortalecimiento de la 
PNH es necesario, además, para frenar la demanda por restablecer las fuerzas armadas en Haití. El 30 de julio de 2008 ex militares se tomaron antiguas dependencias castrenses en demanda por beneficios y por reapertura de las fuerzas armadas. El senador y ex coronel de ejército Youri Latortue, quien preside la comisión senatorial de Justicia y Seguridad, apoya esa causa (International Crisis Group 2008a, Gauthier 2008).

En enero de 2008 se publicaron tres leyes que apuntan a fortalecer el sistema judicial, una que crea el Consejo Superior del Poder Judicial (CSPJ), otra que crea la Academia Judicial y una tercera que regula el estatuto de los magistrados. Sin embargo, su operación depende en gran medida de los donantes internacionales y de la implementación que el Ejecutivo y el parlamento dispongan a través del presupuesto y los nombramientos. El CSPJ aspira a aumentar la independencia del Poder Judicial, actualmente muy limitada, mediante el manejo de la carrera judicial y de su presupuesto. El funcionamiento del CSPJ se ha retrasado porque su presidente es también el presidente de la Corte Suprema, cargo vacante de desde hace cuatro años.

El sistema carcelario también necesita urgentes reformas. Durante el último año varios programas mejoraron las condiciones sanitarias en las principales prisiones, pero el hacinamiento, la falta de seguridad interna y externa de las prisiones, la falta de medidas alternativas de cumplimiento de las sentencias y de sentencias no privativas de libertad para delitos menores, continúan siendo problemas sin solución. El gobierno de Préval no ha dado toda la prioridad a este tema, como prometió a inicios de su mandato (HRW 2008).

Reducir y controlar las bandas de narcotraficantes ha sido uno de los mayores logros del gobierno de Préval, pero es un logro que puede revertirse en cualquier momento. La pobreza, el hacinamiento en las ciudades, el desempleo extremo y la falta de medios básicos de subsistencia son una mezcla potencialmente explosiva en materia de seguridad. Las protestas de abril, así como las de febrero y marzo de 2009, demuestran que el potencial de recrudecimiento de la violencia en una ciudad como Puerto Príncipe, habitada por una población numerosa en situación de extrema pobreza, es una realidad posible de gatillarse en cualquier momento (Feldmann y Montes 2007).

Aún así, las mejoras han sido cuantiosas, al punto que el foco de los esfuerzos internacionales está trasladándose desde lo militar a temas de desarrollo y de policía. Recientemente, Hédi Annabi, Representante Especial del Secretario General de las Naciones Unidas en Haití, comentó al Consejo de Seguridad que "si queremos tener éxito en nuestros esfuerzos, es esencial que haya una mejora en la calidad de vida o, al menos, una esperanza realista de esa mejora". (United Nations News Centre 2009).

\section{ROL DE LA COMUNIDAD INTERNACIONAL: MINUSTAH Y DONANTES}

Haití todavía necesita el apoyo de la comunidad internacional para alcanzar y mantener un punto medio de estabilidad social, económica e institucional que le permita desarrollarse por sí solo y superar los históricos conflictos políticos. El logro de este gobierno, democráticamente electo, debe potenciarse y prolongarse en nuevas experiencias democráticas. Eso no será 
posible sin la presencia constante de la MINUSTAH y de otros organismos internacionales. Las postergadas elecciones de abril de 2009 demostraron que Haití no tiene capacidad de llevar adelante sus procesos eleccionarios sin la ayuda internacional. Otro tanto vale para la reforma de la seguridad, el control fronterizo y la deforestación.

La presencia de la MINUSTAH se justifica al mantener un relativo statu quo en aspectos como la seguridad, democracia y desarrollo. Su sola presencia en el país hace a la nación más atractiva para inversiones internacionales y para el turismo. Muchos cooperantes -sencillamente-no se arriesgarían a invertir en Haití si no hubiera un horizonte de relativa estabilidad. Las protestas de abril se agudizaron por la falta de acción inmediata de la $\mathrm{PNH}$, la UNPOL y las unidades de policía formada (UFPs). La función de la MINUSTAH en materia de seguridad sigue siendo indispensable. La PNH no tiene y no está cerca de tener el personal ni los recursos necesarios para enfrentar por sí sola sus múltiples demandas, las que en países más desarrollados son usualmente satisfechas por varias instituciones: Policías, fuerzas armadas, bomberos, gendarmería, brigadas medioambientales, guardas costeras, etcétera. La PNH simplemente no puede hacer todo lo que se le pide que haga. Con todo, el debate sobre la conversión del carácter militar de la MINUSTAH en uno orientado mayormente a labores de policía y desarrollo, sigue pendiente. Esto en parte por la falta de apoyo de los países latinoamericanos que favorecen la presencia militar más que una policial en Haití. Un paso importante en este sentido fue la inclusión en el mandato de MINUSTAH el control de fronteras, materia en la que se está trabajando.

Ante constantes peticiones del Presidente René Préval, los esfuerzos de la cooperación internacional continúan fluyendo hacia Haití. Dentro de los esfuerzos del Mandatario y de la Primera Ministra Pierre-Louis está asegurar el aporte de los donantes internacionales.

El total de las inversiones de distintos organismos de las Naciones Unidas, por concepto de emergencias en Haití para el año 2008, fue de 16.030.104 dólares estadounidenses (OCHA 2008). Pero la ayuda internacional debe ir más lejos. Durante el 2008 se vio que Haití carece de capacidad para absorber las decenas de haitianos deportados que cada semana llegan desde los EE.UU. y Canadá por la dificultad de regularizar su situación en esos países.

Con proyectos de cooperación internacional de alto impacto y de alta visibilidad se intenta solucionar el problema de la reconstrucción para el desarrollo de Haití, y -si bien- a los esfuerzos militares se ha sumado apoyo en temas de desarrollo, gobernabilidad y control de fronteras, aún parece lejano el momento en que la democracia haitiana se consolide, su economía sea sustentable y se pueda considerar, por lo tanto, una estrategia de salida de la misión extranjera en Haití.

\section{CONCLUSIÓN}

Haití continúa siendo una democracia extremadamente frágil en un Estado con permanente riesgo de desintegración, $\mathrm{o}$-al menos- con capacidades muy precarias de ejercer funciones estatales básicas como el control de sus fronteras, la administración de la Justicia, las funciones de la policía, la selección coordinada de autoridades o la asistencia humanitaria básica. En 
este contexto, el imperio del derecho es naturalmente muy elemental y dispar. Fuera de Puerto Príncipe las capacidades estatales son aún más precarias. Haití depende, y seguirá dependiendo en el futuro previsible de la MINUSTAH y de la cooperación internacional, para mantener su proceso de estabilización política, construcción del Estado, fortalecimiento de la democracia y logro de un desarrollo económico y social sustentable. El gobierno de Préval, gracias al apoyo internacional, ha marcado avances importantes en materia de seguridad, planificación y construcción institucional, pero difícilmente podrá cumplir su mandato y gobernar sin el apoyo internacional, el que se espera que continúe.

Para el 2009 -un año de elecciones senatoriales y locales, y en el que hay reformas constitucionales agendadas, las que darán pie a conflictos entre el gobierno y la oposición, además de los múltiples desafíos provenientes de la naturaleza y la pobreza-controlar la inestabilidad política, social, económica e institucional sigue siendo el principal desafío.

\section{REFERENCIAS}

Banco Interamericano del Desarrollo. 2008. "Remmitances to Haiti topped US\$ 1.65 billion". [En línea] http://www.iadb.org/news/detail.cfm?langauage=English\&id=3637. [Consulta: 05- 04- 2009].

Banco Mundial. 2007. World Development Indicators: Haiti. [En línea] http:/ /web.worldbank.org/haiti. [Consulta: 20-04-2009].

Brea, Jennifer. 2008. "Michèle Pierre-Louis, Haiti's New Prime Minister". Global Voices. August $9^{\text {th }}$. [En línea] http://globalvoicesonline.org/2008/08/09/michele-pierre-louis-haitis-new-prime-minister/. [Consulta: 05-04-2009].

British Broadcasting Corporation. 2008a. "Haiti Storm Damage 'Eye' Popping". October 23 ${ }^{\text {rd }}$. [En línea] http://news.bbc.co.uk/2/hi/americas/7685844.stm. [Consulta: 05-04-2009].

2008b. "Haitian Leader Demand End to Riots". April 9 ${ }^{\text {th }}$. [En línea] http://news.bbc.co.uk/2/ hi/americas/7339755.stm. [Consulta: 10-05-2009].

2008c. "Food Riots Turn Deadly in Haiti". April 5 ${ }^{\text {th }}$ [En línea] http://news.bbc.co.uk/2/hi/ americas/7331921.stm. [Consulta: 10-05-2009].

Charles, Jacqueline. 2009. "Jean-Bertrand Aristide remains potent force in Haiti." The Miami Herald; April 30 rd. [En línea] http://www.miamiherald.com/news/americas/haiti/story/928042.html. [Consulta: 19-05-2009].

Cepal. 2008. "Estudio económico de América Latina 2007-2008". [En línea] http:/ /www.eclac.cl/cgi-bin/ getProd.asp?xml=/publicaciones/xml/9/33869/P33869.xml\&xsl=/de/tpl/p9f.xsl\&base=/de/tpl/ top-bottom.xsl. [Consulta: 19-05-2009].

Coordination Nationale de la Sécurité Alimentaire. 2008. Alerte a l'insécurité Alimentaire. [En línea] http://www.cnsahaiti.org. [Consulta: 05-05-2009].

Dahl, Robert. 1972. Polyarchy: Participation and Opposition. New Haven: Yale University Press.

Feldmann, Andreas; Montes, Esteban. 2008. "Haití: Tribulaciones de un Estado Colapsado". Revista de Ciencia Política UC 28 (1): 245-264.

Gauthier, Amélie. 2008. "Haiti: empty stomachs, stormy politics" Open Democracy Bulletin. [En línea] http://www.opendemocracy.net/article/globalisation/institutions_government/haiti_empty_ stomachs_stormy_politics. [Consulta: 03- 05- 2009].

Human Rights Watch. 2009. World Report 2009, Haiti. [En línea] http://www.hrw.org/en/node/79214. [Consulta: 04-05-2009].

Inter American Dialogue. 2007. "Haiti Real Progress, Real Fragility". [En línea] http:/ /www.the dialogue. org/page.cfm?pageID=115. [Consulta: 04-05-2009].

International Crisis Group. 2009a. "Haiti: Saving the Environment, Preventing Instability and Conflict". [En línea] http://www.crisisgroup.org/library/documents/latin_america/aiti__saving_the_ environment_preventing_instability_and_conflict.pdf, [Consulta: 20-05-2009]. 
. 2009b. "Haiti 2009: Stability at Risk". Latin America and Caribbean Briefing N 19. March. [En línea] http://www.crisisgroup.org/library/documents/latin_america/b19_haiti_2009__stability_ at_risk.pdf. [Consulta: 28-04-2009].

2008a. "Reforming Haiti's security sector". Latin America / Caribbean Report $N^{\circ} 28$. September. [En línea] http:/ / www.crisisgroup.org/home/index.cfm?l=1\&id=5681. [Consulta: 15-05-2009].

. 2008b. "In the Aftermath of Hurricanes, Haiti Situation is Critical". World Politics Review. October. [En línea] http:/ / www.crisisgroup.org/home/index.cfm?id=5739\&]=1. [Consulta: 28-04-2009].

Louidor, Wooldy Edson. 2009. "Tres años de la victoria de René Préval. ¿Dónde estamos? Agencia Latinoamericana de Información. Febrero 6. [En línea] http://alainet.org/active/28860\&lang=es. [Consulta: 28-04-2009].

Mainwaring, Scout y Timothy Scully, eds. 1995. "Building Democratic Institutions. Party Systems in Latin America". Stanford: Stanford University Press.

Médecines Sans Frontièrs. 2008. "Haiti: MSF teams finds villages completely flooded and population stranded with no help one month after cyclones". [En línea] http:/ /www.msf.org/msfinternational/invoke. $\mathrm{cfm}$ ?objectid=C1AD2658-15C5-F00A-251C9E1E8EB25050\&component=toolkit.pressrelease\&method=full_ html. [Consulta: 28-04-2009].

Ministère de la Planification et la Cooperation Externe du Haiti. 2009. "Document de Stratégie Nationale pour la Croissance et pour la réduction de la pauvreté". [En línea] http:/ / www.mpce.gouv.ht/dsrp. htm. [Consulta: 28-04-2009].

Pierre, Hyppolite. 2009. "Reply to Lance Durban's ¿Where is Haiti's Obama?”. Institute for Research in Social Sciences and Politics. [En línea] http:/ /irsp.org/?p=34. [Consulta: 15-05-2009].

Pina, Kevin. 2009. “Controversial Senate Election in Haiti”. Haiti Action. [En línea] http:/ / www.haitiaction. net/News/HIP/4_6_9/4_6_9.html. [Consulta: 20-05-2009].

Programa de Naciones Unidas para el Desarrollo. 2008. Índice de Desarrollo Humano. [En línea] http:/ / hdr.undp.org/es/. [Consulta: 15-05-2009].

Shamsie, Yasmine; Thompson, Andrew. 2006. "Haiti: Hope for a Fragile State". Waterloo: Wilfrid Laurier Press.

UN Office for the Coordination of Humanitarian Affairs. 2008. United Central Emergency Response Fund. [En línea] http:/ / ochaonline.un.org/Default.aspx?tabid=4629. [Consulta: 15-05-2009].

United Nations News Centre. 2009. "International donors' conference could spur Haiti's recovery, says Ban". April 13. [En línea] http://www.un.org/apps/news/story.asp?NewsID=30460\&Cr=haiti\&Cr1=. [Consulta: 15-05-2009]. 
